\title{
A CHARACTERIZATION OF HARDY-ORLICZ SPACES ON PLANAR DOMAINS
}

\author{
MANFRED STOLL
}

(Communicated by Theodore W. Gamelin)

\begin{abstract}
In the paper we prove that for a wide class of bounded domains $D$ in $\mathbb{C}$, a holomorphic function $f$ is in the Hardy-Orlicz space $H_{\phi}(D)$ if and only if

$$
\iint_{D} \delta(z) \phi^{\prime \prime}(\log |f(z)|) \frac{\left|f^{\prime}(z)\right|^{2}}{|f(z)|^{2}} d x d y<\infty,
$$

where $\delta(z)$ denotes the distance from $z$ to the boundary of $D$ and $\phi$ is a strongly convex function on $(-\infty, \infty)$ for which $\phi^{\prime \prime}(t)$ exists for all $t$.
\end{abstract}

\section{INTRODUCTION}

For $0<p<\infty$, the Hardy classes $H^{p}$ in the unit disc $U=\{z \in \mathbb{C}:|z|<1\}$ are defined as the set of holomorphic functions $f$ in $U$ for which

$$
\sup _{0<r<1} \int_{0}^{2 \pi}\left|f\left(r e^{i \theta}\right)\right|^{p} d \theta<\infty .
$$

It is well known that this is equivalent to the subharmonic function $|f(z)|^{p}$ having a harmonic majorant on $U$. In [Y1] Yamashita proved that a holomorphic function $f$ on $U$ is in $H^{p}, 0<p<\infty$, if and only if

$$
\iint_{U}(1-|z|)|f(z)|^{p-2}\left|f^{\prime}(z)\right|^{2} d x d y<\infty .
$$

The case $p=2$ had previously been noted in [Z, Remark(a), p. 208]. Analogous problems have also been considered by Kobayashi and Suita in [KS] for $H^{p}$ spaces on a nonparabolic Riemann surface $R$. In a subsequent paper [Y2] Yamashita proved that if $f \in H^{p}$, then

$$
\lim _{r \rightarrow 1}(1-r) \iint_{|z| \leq r}|f(z)|^{p-2}\left|f^{\prime}(z)\right|^{2} d x d y=0 .
$$

The purpose of this note is to obtain analogous results for functions in the Hardy-Orlicz class $H_{\phi}$ on planar domains in $\mathbb{C}$ that have a Green's function $G$. For convenience we will take the Green's function to be positive and hence superharmonic.

Received by the editors March 11, 1991 and, in revised form, July 18, 1991.

1991 Mathematics Subject Classification. Primary 30D55. 
As in [R2], a function $\phi$ on $(-\infty, \infty)$ is said to be strongly convex if $\phi$ is nonnegative, convex, and nondecreasing with $\phi(t) / t \rightarrow \infty$ as $t \rightarrow \infty$. Let $D$ be any domain in $\mathbb{C}$. A holomorphic function $f$ on $D$ is said to be in the Hardy-Orlicz class $H_{\phi}(D)$ if there exists a harmonic function $h$ on $D$ such that

$$
\phi(\log |f(z)|) \leq h(z)
$$

for all $z \in D$. Taking $\phi(t)=e^{p t}$ gives the usual Hardy space $H^{p}(D)$ as considered in [R1]. The main result of the paper is

Theorem. Let $D$ be a domain in $\mathbb{C}$ that has a Green's function $G$, and let $\phi$ be a strongly convex function such that $\phi^{\prime \prime}(t)$ exists for all $t \in \mathbb{R}$. A holomorphic function $f \in H_{\phi}(D)$ if and only if

$$
\iint_{D} G\left(t_{o}, z\right) \phi^{\prime \prime}(\log |f(z)|) \frac{\left|f^{\prime}(z)\right|^{2}}{|f(z)|^{2}} d x d y<\infty
$$

for some $t_{o} \in D$. Futhermore, if $f \in H_{\phi}(D)$ then

$$
\lim _{\varepsilon \rightarrow 0} \varepsilon \iint_{\Omega_{\varepsilon}} \phi^{\prime \prime}(\log |f(z)|) \frac{\left|f^{\prime}(z)\right|^{2}}{|f(z)|^{2}} d x d y=0,
$$

where $\Omega_{\varepsilon}=\left\{z \in D: G\left(t_{o}, z\right) \geq \varepsilon\right\}$.

As a consequence of the theorem, we will show that for a wide class of bounded domains $D$, a holomorphic function $f \in H_{\phi}(D)$ if and only if

$$
\iint_{D} \delta(z) \phi^{\prime \prime}(\log |f(z)|) \frac{\left|f^{\prime}(z)\right|^{2}}{|f(z)|^{2}} d x d y<\infty,
$$

where $\delta(z)$ denotes the distance from $z$ to the boundary of $D$. Furthermore, if $f \in H_{\phi}$ then

$$
\lim _{s \rightarrow 0} s \iint_{D_{s}} \phi^{\prime \prime}(\log |f(z)|) \frac{\left|f^{\prime}(z)\right|^{2}}{|f(z)|^{2}} d x d y=0,
$$

where $D_{s}=\{z \in D: \delta(z) \geq s\}$. For the unit disc $U, \delta(z)=(1-|z|)$, and thus (1.6) and (1.7) with $\phi(t)=e^{p t}$ imply the results of Yamashita.

If $f$ is holomorphic in $D$ and $\phi$ is strongly convex, then the function $g(z)=\phi(\log |f(z)|)$ is a nonnegative subharmonic function on $D$. In $\S 2$ we will use potential theory methods to obtain a characterization of those subharmonic functions $f$ on $D$ that have a harmonic majorant in terms of the Riesz measure $\mu_{f}$ of $f$. The theorem is proved in $\S 3$ by computing the Riesz measure of $g(z)=\phi(\log |f(z)|)$. In $\S 4$ we also give a characterization of the Nevanlinna class $N(D)$ and indicate how the results can be extended to Hardy-Orlicz spaces $H_{\phi}$, where $\phi(\log |f(z)|)=\phi\left(\log ^{+}|f(z)|\right)$.

\section{HARMONIC MAJORANTS OF SUBHARMONIC FUNCTIONS}

For a domain $D$ in $\mathbb{C}$, we denote by $S(D)$ the class of subharmonic functions $f$ on $D$ for which $f$ has a harmonic majorant on $D$. It is well known that if a subharmonic function $f$ has a harmonic majorant on $D$, then it has a least harmonic majorant on $D$. For $f \in S(D)$, we denote the least harmonic 
majorant of $f$ by $h_{f}$. In the definition of subharmonic we exclude the case $f \equiv-\infty$.

Let $f$ be subharmonic on $D$. The Riesz measure of $f$ is the nonnegative regular Borel measure $\mu_{f}$ on $D$ satisfying

$$
\iint_{D} \varphi d \mu_{f}=\frac{1}{2 \pi} \iint_{D} f \nabla^{2} \varphi d \lambda
$$

for all $\varphi \in C_{c}^{\infty}(D)$, where $\nabla^{2} \varphi$ denotes the Laplacian of $\varphi$ and $\lambda$ is twodimensional area measure on $D$. If $f$ is $C^{2}$, then by Green's identity, $d \mu_{f}=$ $\frac{1}{2 \pi} \nabla^{2} f d \lambda$.

By a regular exhaustion $\left\{\Omega_{n}\right\}$ of $D$ we mean a sequence of domains $\Omega_{n}$, with compact boundary $\Gamma_{n}$ such that $\Omega_{n} \cup \Gamma_{n} \subset \Omega_{n+1}, \cup_{1}^{\infty} \Omega_{n}=D$, and such that the Dirichlet problem is solvable for $\Omega_{n}$. By [F, Proposition 1.5.3], each domain in $\mathbb{C}$ has a regular exhaustion. For each $n$, let $G_{n}$ denote the Green's function of $\Omega_{n}$. If $f$ is subharmonic on $D$ with Riesz measure $\mu_{f}$, then by the Riesz Decomposition Theorem

$$
f(z)=-\iint_{\Omega_{n}} G_{n}(z, w) d \mu_{f}(w)+h_{n}(z),
$$

for all $z \in \Omega_{n}$, where $h_{n}(z)$ is the least harmonic majorant of $f$ on $\Omega_{n}$. If the boundary $\Gamma_{n}$ of $\Omega_{n}$ consists of a finite number of disjoint simple closed analytic curves, then

$$
h_{n}(z)=\frac{1}{2 \pi} \int_{\Gamma_{n}} f(t) \frac{\partial G_{n}}{\partial \eta}(z, t) d s(t),
$$

where the normal derivative of $G_{n}$ is taken along the interior normal $\eta$. For proofs of the above the reader is referred to [T].

The following proposition, which undoubtedly has been proved elsewhere, is included for completeness.

Proposition 1. Let $f$ be subharmonic in $D$ and let $\mu_{f}$ be the Riesz measure of $f$. Then $f \in S(D)$ if and only if there exists $t_{o} \in D$ such that

$$
\sup _{n} \iint_{\Omega_{n}} G_{n}\left(t_{o}, z\right) d \mu_{f}(z)<\infty
$$

for any regular exhaustion $\left\{\Omega_{n}\right\}$ of $D$. If the domain $D$ has a Green's function $G$, then $f \in S(D)$ if and only if there exists $t_{o} \in D$ such that

$$
\iint_{D} G\left(t_{o}, z\right) d \mu_{f}(z)<\infty
$$

Proof. Suppose $f \in S(D)$. Let $h$ be any harmonic majorant of $f$ on $D$, and let $\left\{\Omega_{n}\right\}$ be a regular exhaustion of $D$. For each $n$, let $h_{n}$ be the least harmonic majorant of $f$ on $\Omega_{n}$ given by (2.2). Since $f \leq h$ on $\Omega_{n}$, we have that $h_{n}(z) \leq h(z)$ for all $z \in \Omega_{n}$. Since $f(z)>-\infty$ a.e. on $D$, there exists $t_{o} \in \Omega_{1}$ such that $f\left(t_{o}\right)>-\infty$. For this $t_{o}$, by (2.2),

$$
\iint_{\Omega_{n}} G_{n}\left(t_{o}, z\right) d \mu_{f}(z)=h_{n}\left(t_{o}\right)-f\left(t_{o}\right) \leq h\left(t_{o}\right)-f\left(t_{o}\right),
$$

which proves (2.4). If the domain $D$ itself has a Green's function $G$, then $G_{n}\left(t_{o}, z\right) \leq G\left(t_{o}, z\right)$ for all $z \in \Omega_{n}$ and $G\left(t_{o}, z\right)=\lim _{n \rightarrow \infty} G_{n}\left(t_{o}, z\right)$. Thus (2.5) follows by the Monotone Convergence Theorem. 
Conversely, suppose (2.4) holds for some $t_{o} \in D$ and any regular exhaustion $\left\{\Omega_{n}\right\}$ of $D$. Without loss of generality we may assume $t_{o} \in \Omega_{n}$ for all $n$. For each $n$ let $h_{n}$ be the least harmonic majorant of $f$ on $\Omega_{n}$ as given by (2.2). Clearly, if $n<m$, then $h_{n}(z) \leq h_{m}(z)$ for all $z \in \Omega_{n}$. By hypothesis, there exists a constant $M$ such that $h_{n}\left(t_{o}\right) \leq M+f\left(t_{o}\right)$ for all $n$. Hence by Harnack's Theorem, $h(z)=\lim _{n \rightarrow \infty} h_{n}(z)$ is harmonic on $D$, and thus $f \in S(D)$.

Let $D$ be a domain with Green's function $G$. For $t \in D$ and $\varepsilon>0$, let

$$
\Omega_{\varepsilon}(t)=\{z \in D: G(t, z) \geq \varepsilon\} .
$$

Proposition 2. Let $D$ be a domain with Green's function $G$, and let $f \in S(D)$ with Riesz measure $\mu_{f}$. Then

$$
\lim _{\varepsilon \rightarrow 0^{+}} \varepsilon \mu_{f}\left(\Omega_{\varepsilon}(t)\right)=0 \quad \text { for all } t \in D .
$$

Proof. Let $t_{o} \in D$ be arbitrary and set

$$
g(z)=\iint_{D} G(z, w) d \mu_{f}(w) .
$$

Since $s \rightarrow \mu_{f}\left(\Omega_{s}\left(t_{o}\right)\right)$ is the distribution function of $G\left(t_{o}, w\right)$,

$$
\int_{0}^{\infty} \mu_{f}\left(\Omega_{s}\left(t_{o}\right)\right) d s=\iint_{D} G\left(t_{o}, w\right) d \mu_{f}(w)=g\left(t_{o}\right) .
$$

Therefore,

$$
\varepsilon \mu_{f}\left(\Omega_{\varepsilon}\left(t_{o}\right)\right) \leq \int_{0}^{\varepsilon} \mu_{f}\left(\Omega_{s}\left(t_{o}\right)\right) d s \rightarrow 0 \quad \text { as } \varepsilon \rightarrow 0
$$

whenever $g\left(t_{o}\right)<\infty$. When $g\left(t_{o}\right)=\infty$, choose $\alpha$ sufficiently large so that $\Omega_{\alpha}\left(t_{o}\right)$ is compact, and let $\nu_{\alpha}$ be the measure defined by $\left(1-\chi_{\alpha}\right) \mu_{f}$, where $\chi_{\alpha}$ is the characteristic function of $\Omega_{\alpha}\left(t_{o}\right)$. Let $g_{\alpha}$ denote the corresponding function defined by (2.7) for the measure $\nu_{\alpha}$. Since $g_{\alpha}$ is harmonic at $t_{o}$, it follows that $g_{\alpha}\left(t_{o}\right)<\infty$, and hence as above, $\varepsilon \nu_{\alpha}\left(\Omega_{\varepsilon}\left(t_{o}\right)\right) \rightarrow 0$ as $\varepsilon \rightarrow 0$. Since $\mu_{f}\left(\Omega_{\alpha}\left(t_{o}\right)\right)$ is finite and

$$
\varepsilon \mu_{f}\left(\Omega_{\varepsilon}\left(t_{o}\right)\right)=\varepsilon \mu_{f}\left(\Omega_{\alpha}\left(t_{o}\right)\right)+\varepsilon \nu_{\alpha}\left(\Omega_{\varepsilon}\left(t_{o}\right)\right),
$$

$\lim _{\varepsilon \rightarrow 0} \varepsilon \mu_{f}\left(\Omega_{\varepsilon}\left(t_{o}\right)\right)=0$, which proves the result.

We now assume that $D$ is a bounded domain in $\mathbb{C}$ with Green's function $G$. For $z \in D$, let $\delta(z)$ denote the distance from $z$ to $\partial D$, the boundary of $D$. We will assume that the Green's function $G$ satisfies the following: for each $t_{o} \in D$, there exist constants $c_{1}$ and $c_{2}$, depending only on $t_{o}$ and $D$, such that

$$
\begin{array}{ll}
\text { (i) } c_{1} \delta(z) \leq G\left(t_{o}, z\right) & \text { for all } z \in D, \\
\text { (ii) } G\left(t_{o}, z\right) \leq c_{2} \delta(z) & \text { for all } z \in D \sim D\left(t_{o}, \frac{1}{2} \delta\left(t_{o}\right)\right) \text {. }
\end{array}
$$

In the case where the boundary of $D$ consists of a finite number of disjoint, simple, closed analytic curves, (2.8) was proved in [W]. If the boundary of $D$ is $C^{2}$, then the inequalities can be established by comparing the Green's function $G$ to the Green's function of discs which are internally tangent to obtain (i) and to the Green's function of the complement of externally tangent discs to obtain (ii). 
Proposition 3. Let $D$ be a bounded domain with Green's function $G$ satisfying (2.8), and let $f$ be subharmonic on $D$ with Riesz measure $\mu_{f}$. Then $f \in S(D)$ if and only if

$$
\iint_{D} \delta(z) d \mu_{f}(z)<\infty .
$$

Furthermore, if $f \in S(D)$, then

$$
\lim _{s \rightarrow 0^{+}} s \mu_{f}\left(D_{s}\right)=0,
$$

where $D_{s}=\{z \in D: \delta(z) \geq s\}$.

Proof. Suppose $f \in S(D)$ and $t_{o} \in D$ is such that (2.5), and hence also (2.6), holds. Then (2.9) follows by (2.8(i)), and since $D_{s} \subset \Omega_{c_{1} s}\left(t_{o}\right)$, we obtain (2.10).

Conversely, suppose (2.9) holds. Let $p_{o} \in D$ be arbitrary and let $K=$ $\bar{D}\left(p_{o}, \frac{1}{2} \delta\left(p_{o}\right)\right)$. Let

$$
g_{1}(z)=\iint_{K} G(z, w) d \mu_{f}(w) .
$$

Since $K$ is compact, $g_{1}$ is superharmonic on $D$. In particular, $g_{1}(z)<\infty$ a.e. on $D$. Also, let

$$
g_{2}(z)=\iint_{D \sim K} G(z, w) d \mu_{f}(w) .
$$

Since $G\left(p_{o}, w\right) \leq c_{2} \delta(w)$, we have $g_{2}\left(p_{o}\right)<\infty$ by hypothesis. Thus $g_{2}(z)$ is also superharmonic on $D$ and hence finite a.e. Thus there exists $t_{o} \in D$ such that both $g_{1}$ and $g_{2}$ are finite at $t_{o}$. Therefore $\mu_{f}$ satisfies (2.5), which proves the result.

\section{Proof of the theorem}

Let $D$ be a domain in $\mathbb{C}$ with Green's function $G$. Suppose $f \in H_{\phi}(D)$, where $\phi$ is a strongly convex function for which $\phi^{\prime \prime}(t)$ exists for all $t \in \mathbb{R}$. Since $\phi$ is nonnegative and nondecreasing, $\phi(-\infty)=\lim _{t \rightarrow-\infty} \phi(t)$ exists. Also, since $\phi^{\prime \prime}(t)$ exists for all $t \in \mathbb{R}$ and $\phi^{\prime \prime}(t) \geq 0$, it follows that $\phi^{\prime}(t)$ is continuous, nondecreasing, and nonnegative on $\mathbb{R}$. As a consequence, $\lim _{t \rightarrow-\infty} \phi^{\prime}(t)$ exists. Furthermore, since $\phi(t)=\phi(-\infty)+\int_{-\infty}^{t} \phi^{\prime}(x) d x$, we have

$$
\lim _{t \rightarrow-\infty} \phi^{\prime}(t)=0 \text {. }
$$

Let $g(z)=\phi(\log |f(z)|)$. Then $g$ is a nonnegative subharmonic function on $D$. To prove the theorem it suffices to show that the Riesz measure $\mu_{g}$ is absolutely continuous and is given by

$$
d \mu_{g}(z)=\frac{1}{2 \pi} \phi^{\prime \prime}(\log |f(z)|) \frac{\left|f^{\prime}(z)\right|^{2}}{|f(z)|^{2}} d \lambda(z) .
$$

For convenience, set

$$
f_{\phi}^{\sharp}(z)=\phi^{\prime \prime}(\log |f(z)|) \frac{\left|f^{\prime}(z)\right|^{2}}{|f(z)|^{2}} .
$$


Let $D_{o}=\{z \in D: f(z)=0\}$. By a routine computation, on $D \sim D_{o}$, $\nabla^{2} g(z)=f_{\phi}^{\sharp}(z)$. Thus to show that $\mu_{g}$ is absolutely continuous and is given by the above, it suffices to show that

$$
\iint_{D} \varphi f_{\phi}^{\sharp} d \lambda=\iint_{D} g \nabla^{2} \varphi d \lambda
$$

for all $\varphi \in C_{c}^{\infty}(D)$.

Suppose $\varphi \in C_{c}^{\infty}(D)$. Since $\varphi$ has compact support and the zeros of $f$ are isolated, we can assume without loss of generality that the support of $\varphi$ contains exactly one zero of $f$, say $p_{o}$, which for convenience we will take to be zero. Let $r>0$ be such that $\bar{D}(0, r) \subset D$ and that $f(z) \neq 0$ for all $z \in \bar{D}(0, r), z \neq 0$. Let $D_{r}=D \sim \bar{D}(0, r)$ and let $S_{r}=\partial D(0, r)$. Then by Green's identity,

$$
\iint_{D_{r}}\left[\varphi \nabla^{2} g-g \nabla^{2} \varphi\right] d \lambda=\int_{S_{r}}\left[\varphi \frac{\partial g}{\partial \eta}-g \frac{\partial \varphi}{\partial \eta}\right] d s .
$$

We now show that the term on the right goes to zero as $r \rightarrow 0$. Since $\frac{\partial \varphi}{\partial \eta}$ and $g\left(r e^{i \theta}\right)$ are bounded in a neighborhood of 0 , we have

$$
\lim _{r \rightarrow 0} \int_{S_{r}} g \frac{\partial \varphi}{\partial \eta} d s=0
$$

Consider

$$
\int_{S_{r}} \varphi \frac{\partial g}{\partial \eta} d s=-r \int_{0}^{2 \pi} \varphi\left(r e^{i \theta}\right) \frac{\partial}{\partial r} \phi\left(\log \left|f\left(r e^{i \theta}\right)\right|\right) d \theta
$$

But $\frac{\partial}{\partial r} \phi\left(\log \left|f\left(r e^{i \theta}\right)\right|\right)=\phi^{\prime}\left(\log \left|f\left(r e^{i \theta}\right)\right|\right) \frac{\partial}{\partial r} \log \left|f\left(r e^{i \theta}\right)\right|$. Suppose $f$ has a zero of order $m \geq 1$ at 0 . Then $f(w)=w^{m} h(w)$ where $h$ is holomorphic in a neighborhood of 0 with $h(0) \neq 0$. Therefore $\log \left|f\left(r e^{i \theta}\right)\right|=m \log r+$ $\log \left|h\left(r e^{i \theta}\right)\right|$, and thus

$$
\frac{\partial}{\partial r} \log \left|f\left(r e^{i \theta}\right)\right|=\frac{m}{r}+O(1)
$$

Therefore

$$
\left|\varphi\left(r e^{i \theta}\right) \frac{\partial}{\partial r} \phi\left(\log \left|f\left(r e^{i \theta}\right)\right|\right)\right| \leq \frac{M}{r} \phi^{\prime}\left(\log \left|f\left(r e^{i \theta}\right)\right|\right) .
$$

By $(3.1), \phi^{\prime}\left(\log \left|f\left(r e^{i \theta}\right)\right|\right) \rightarrow 0$ as $r \rightarrow 0$. Thus

$$
\lim _{r \rightarrow 0}\left|\int_{S_{r}} \varphi \frac{\partial g}{\partial \eta} d s\right|=0
$$

Finally, since $\varphi$ has compact support and $g$ is bounded on the support of $\varphi$,

$$
\lim _{r \rightarrow 0} \iint_{D_{r}} g \nabla^{2} \varphi d \lambda=\iint_{D} g \nabla^{2} \varphi d \lambda .
$$

Therefore, $\lim _{r \rightarrow 0} \iint_{D_{r}} \varphi f_{\phi}^{\sharp} d \lambda$ exists and is finite, and as a consequence, (3.3) holds for all $\varphi \in C_{c}^{\infty}(D)$. Therefore $\mu_{g}$ is absolutely continuous and is given by (3.2). 
Remark. If the Green's function $G$ of $D$ satisfies (2.8), then $f \in H_{\phi}(D)$ if and only if (1.6) holds and if this is the case then (1.7) holds also.

\section{RelATED RESUltS}

The methods of the preceding section are not restricted to the Hardy-Orlicz spaces $H_{\phi}$ where $\phi^{\prime \prime}(t)$ exists for all $t$. They can also be applied to other spaces of holomorphic functions, including the Nevanlinna class $N(D)$ and Hardy-Orlicz spaces $H_{\phi}$, where $\phi(\log |f(z)|)=\phi\left(\log ^{+}|f(z)|\right)$ and $\phi^{\prime \prime}$ does not exist at 0 . As in [R1], let $\log ^{+}(D)$ denote the set of holomorphic functions $f$ on $D$ for which $\log ^{+}|f(z)|=\max \{\log |f(z)|, 0\}$ has a harmonic majorant on $\mathrm{D}$. The space $\log ^{+}(D)$ is also referred to as the Nevanlinna class $N(D)$. From the inequality

$$
\log ^{+} x \leq \log (1+x) \leq \log ^{+} x+\log 2 \quad(x \geq 0),
$$

$f \in \log ^{+}(D)$ if and only if the subharmonic function $g(z)=\log (1+|f(z)|)$ is in $S(D)$.

As in $\S 3$, let $D_{o}=\{z \in D: f(z)=0\}$. Then on $D \sim D_{o}$,

$$
\nabla^{2} g(z)=\frac{1}{(1+|f(z)|)^{2}} \frac{\left|f^{\prime}(z)\right|^{2}}{|f(z)|} .
$$

If $p_{o}$ is a zero of $f$ of order $m$, then in a neighborhood of $p_{o}$,

$$
g\left(p_{o}+r e^{i \theta}\right)=\log \left(1+r^{m}\left|h\left(p_{o}+r e^{i \theta}\right)\right|\right),
$$

where $h$ is analytic at $p_{o}$ with $h\left(p_{o}\right) \neq 0$. Hence for $r$ sufficiently small, $\left|\frac{\partial}{\partial r} g\left(p_{o}+r e^{i \theta}\right)\right|=O\left(r^{m-1}\right)$, and consequently,

$$
\left|\int_{S_{r}} \varphi \frac{\partial g}{\partial \eta} d s\right|=O\left(r^{m}\right)
$$

Thus, as in $\S 3$,

$$
d \mu_{g}(z)=\frac{1}{2 \pi} \frac{1}{(1+\mid f(z \mid))^{2}} \frac{\left|f^{\prime}(z)\right|^{2}}{|f(z)|} d \lambda(z) .
$$

As a consequence we obtain

Proposition 4. Let $D$ be a domain with Green's function $G$. Then $f \in \log ^{+}(D)$ if and only if

$$
\iint_{D} G\left(t_{o}, z\right) \frac{\left|f^{\prime}(z)\right|^{2}}{(1+|f(z)|)^{2}|f(z)|} d \lambda(z)<\infty
$$

for any $t_{o} \in D$.

The methods can also be extended to Hardy-Orlicz spaces where the strongly convex function $\phi$ satisfies

(1) $\phi(t)=0$ for all $t<0$ with $\phi(0)=\phi^{\prime}(0)=0$,

(2) $\phi^{\prime \prime}(t)$ exists for all $t>0$, and

(3) $\phi(2 t) \leq c \phi(t)$ for some positive constant $c$ and all $t>0$.

The function $\phi_{\alpha}$ defined for $\alpha>1$ by $\phi_{\alpha}(t)=t^{\alpha}$ for $t>0$ and $\phi_{\alpha}(t)=0$ for $t \leq 0$ is an example of such a function. By property (3) and equation (4.1), $f \in H_{\phi}(D)$ if and only if the subharmonic function $g(z)=\phi(\log (1+|f(z)|))$ 
has a harmonic majorant on $D$. As in $\S 3$, the Riesz measure $\mu_{g}$ of $g$ is given by

$$
d \mu_{g}(z)=\frac{1}{2 \pi}\left[\phi^{\prime \prime}(\log (1+|f(z)|))+\frac{\phi^{\prime}(\log (1+|f(z)|))}{|f(z)|}\right] \frac{\left|f^{\prime}(z)\right|^{2}}{(1+|f(z)|)^{2}} d \lambda(z) .
$$

\section{ACKNOWLEDGMENT}

The author would like to express his thanks to the referee for noting an error in the author's original proof of Proposition 2 and for suggesting the present proof.

\section{REFERENCES}

[F] S. D. Fisher, Function theory on planar domains, John Wiley \& Sons, New York, 1983.

[KS] S. Kobayashi and N. Suita, Area integrals and $H_{p}$ norms of analytic functions, Complex Variables 5 (1986), 181-188.

[R1] W. Rudin, Analytic functions of class $H_{p}$, Trans. Amer. Math. Soc. 78 (1955), 46-66.

[R2] _ Function theory in polydiscs, W. A. Benjamin, Inc, New York, 1969.

[T] M. Tsuji, Potential theory, Chelsea Publ. Co., New York, 1975.

[W] E. Wojcicka, Functions of bounded characteristic in multiply connected domains, Doctoral Dissertation, Univ. of South Carolina, 1985.

[Y1] S. Yamashita, Criteria for functions to be of Hardy class $H^{p}$, Proc. Amer. Math. Soc. 75 (1979), 69-72.

[Y2] - Holomorphic functions and area integrals, Boll. Un. Math. Ital. A (1) 6 (1982), $115-120$

[Z] A. Zygmund, Trigonometric series, Cambridge Univ. Press, London, 1968.

Department of Mathematics, University of South Carolina, Columbia, South CarOLINA 29208

E-mail address: stoll@milo.math.scarolina.edu 